\title{
Multidimensionality of Delocalization Indices and Nucleus Independent Chemical Shifts in Polycyclic Aromatic Hydrocarbons II: Proof of Further Non-Locality.
}

Stijn Fias, Sofie Van Damme, Patrick Bultinck*

December 7, 2009

\footnotetext{
Department of Inorganic and Physical Chemistry, Ghent University, Krijgslaan 281 (S3), B-9000 Ghent, Belgium.

E-mail: Patrick.Bultinck@UGent.be ; fax:(+32) 926-44-983

E-mail: Stijn.Fias@UGent.be; fax:(+32) 926-44-983
} 


\begin{abstract}
In a recent contribution we examined the effect of ten- and fourteencenter circuits on the Nucleus Independent Chemical Shifts (NICS) using Multicentre Bond Indices $(\mathrm{MCBI})^{[1]}$. In this study the non-local contributions to the NICS are further investigated for a larger set of polycyclic aromatic hydrocarbons (PAH). To achieve this the NICS are predicted using the MCBI and compared with ab initio results. The NICS of the central ring of Perylene- and Benzo-[ghi]perylenelike fragments and of Coronene appear to have other non-local contributions than the ones previously studied. It is shown that a model based on the MCBI-Ring Current maps and the inclusion of new circuits proves the existence and shows the nature of these new non-local effects on the NICS. This new model leads to a better understanding of the differences between the NICS and delocalisation indices. The results show that the NICS value is not only significantly influenced by the higher-order circuits encircling the ring at which it is evaluated, but also by the local aromaticity of the surrounding rings and occasionally, like in the case of Coronene, the NICS are even influenced by currents farther away in the molecule.
\end{abstract}

Keywords: Aromaticity, NICS, Generalized Population Analysis,

Multicenter Bond Index, multidimensionality of aromaticity.

\title{
1 Introduction
}

Few concepts in chemistry are as widespread as aromaticity. Although originally well defined in terms of similarity between benzenoid rings ${ }^{[2]}$, over time 
it has been used for many different classes of molecules, making it a fuzzy concept. The lack of an immediate observable has resulted in a multiplicity of indices to quantify aromaticity, based on energetic ${ }^{[3]}$, geometric ${ }^{[4]}$, quantum chemical ${ }^{[5]}$ or magnetic considerations ${ }^{[6-10]}$. A concept closely related to aromaticity is that of electron delocalization, of which the Multi Centre Bond Index (MCBI), recently introduced by our group ${ }^{[11-14]}$, has been shown to be a successful measure ${ }^{[15]}$. In the family of magnetic measures of aromaticity, one of the most popular indices is the so-called Nucleus Independent Chemical Shift (NICS) ${ }^{[6,7,16,17]}$ which is the negative of the magnetic shielding computed at the centre of the ring for which the degree of aromaticity is to be assessed. The lack of correlation between some of these indices has put forward suggestions that aromaticity is multidimensional phenomenon ${ }^{[3,18-21]}$. Recently many authors started to refer to the local-aromaticity of separate rings in molecules, adding to the confusion around the concept of aromaticity. Local-aromaticity refers to the degree of aromaticity of a fragment of a molecule, typically the degree of aromaticity of a specific benzenoid ring in a polyaromatic hydrocarbon. Local aromaticity of a benzenoid ring will be used here to reflect the retention of Benzene characteristics. Many works implicitly assume that local aromaticity is also a multidimensional concept. However, recent works have shown that much of the lack of correlation between especially MCBI and NICS is not due to such multidimensional character, but rather due to the fact that they reflect different phenomena. In case of molecules with several rings, NICS computed at the center of a specific ring are, often assumed to reflect the local degree of (benzenoid) aromaticity. However, NICS computed in a single point in these molecules reflect contri- 
butions from all circuits in those molecules. MCBI, on the other hand, are a single value that reflect delocalisation in a single circuit. Through statistical analysis, the present authors have previously shown that for a large set of PAH, the NICS values can be derived from MCBI, refuting the need to invoke a local analogue of the multidimensionality of aromaticity. It was also shown that ring current maps derived from MCBI, agree very well with ab initio computed ring current maps ${ }^{[1,22,23]}$.

The model reconciling magnetic and MCBI data relies on the fact that one can consider the total ring current as composed of different circuitcontributons $^{[24-26]}$. For example, Anthracene contains six different aromatic circuits, three benzenoid circuits, two naphthalene like circuits and one circuit that follows the edge of the entire molecule. The ring current $\mathbf{J}(\mathbf{r})$ in every point in the molecule can thus be considered as arising from these different circuits ${ }^{[8]}$. In Anthracene, one can write:

$$
\begin{aligned}
\mathbf{J}(\mathbf{r}) & =\mathbf{J}_{B e n z 1}(\mathbf{r})+\mathbf{J}_{B e n z 2}(\mathbf{r})+\mathbf{J}_{B e n z 3}(\mathbf{r}) \\
& +\mathbf{J}_{\text {Naph } 1}(\mathbf{r})+\mathbf{J}_{N a p h 2}(\mathbf{r}) \\
& +\mathbf{J}_{\text {Edge }}(\mathbf{r})
\end{aligned}
$$

Analogous to equation 1, the authors suggested that the NICS originates from all circuits in a molecule, or once again for Anthracene: 


$$
\begin{aligned}
\operatorname{NICS}(\mathbf{r}) & =\operatorname{NICS}_{b e n z 1}(\mathbf{r})+\operatorname{NICS}_{b e n z 2}(\mathbf{r})+\operatorname{NICS}_{b e n z 3}(\mathbf{r}) \\
& +\operatorname{NICS}_{\text {naph } 1}(\mathbf{r})+\operatorname{NICS}_{\text {naph } 2}(\mathbf{r}) \\
& +\operatorname{NICS}_{\text {edge }}(\mathbf{r})
\end{aligned}
$$

Supposing that for each circuit the NICS contribution and the multicenter index correlate and that the contribution of circuits that do not encircle the point at which the NICS is calculated is negligible ${ }^{[27]}$, the total NICS at the centre of a given six-membered ring $\mathrm{X}$ can be expressed as a linear combination of the six-centre index (SCI), the Naphthalene-like ten-centre indices (TCI) and the Anthracene- and Phenanthrene-like fourteen-centre indices (FCI-a and FCI-b) for the circuits that include $\mathrm{X}^{[1]}$ :

$$
\operatorname{NICS}\left(\mathbf{r}_{\mathrm{x}}\right)=a \mathrm{SCI}+b \sum_{j} \mathrm{TCI}_{j}+c \sum_{k}\left(\mathrm{FCI}-\mathrm{a}_{k}+\mathrm{FCI}^{-\mathrm{b}_{k}}\right)+d
$$

The NICS were fitted using this equation and constants a, b, c, and d were calculated. The ab initio NICS-values and the NICS predicted by the model are significantly correlated with $\mathrm{R}^{2}=0.84$ and above. The lack of correlation $\left(\mathrm{R}^{2}=0.38\right)$ between NICS computed at a benzenoid ring center and the multicenter index for the benzenoid circuit, without the higher-order circuits, demonstrates that NICS should not be used to assess the degree of local aromaticity of a benzenoid circuit. The significant correlations found between the NICS and the MCBI when including the higher-order circuits imply that there is a significant influence of these different circuits on the NICS, in addition to the local (benzenoid) aromaticity. Therefore, it should be avoided to report correlations between aromaticity indices reflecting only 
a specific circuit and the NICS values, as the latter are influenced by all other circuits.

In a different study ${ }^{[22]}$ it was shown that MCBI delocalization indices and Ring Current(RC) maps can be reconciled using a similar reasoning. Approximate Ring Current maps have been constructed from the MCBI by projecting the SCI as vectors on the bonds of the benzenoid ring in a diatropic manner. This leads to the partial or full cancellation of the SCI vectors of two adjoint benzenoid rings on their common bond. The same was done for the ten-, and fourteen-centre indices (TCI, FCI). The parameters obtained from the regression in equation 3 were used as weighting factors to scale and sum the SCI-, TCI- and FCI-vector maps to the final MCBI-RC map. The MCBI-current maps and ab initio ring current maps were calculated and compared for $394 \mathrm{PAH}$ and they were visually confirmed to be the same for all molecules. The results prove that the MCBI contain the necessary information for the prediction of current density maps in $\mathrm{PAH}$.

In the present paper we further study the non-local contributions to the NICS by testing the correlation between the MCBI and the NICS using the method in equation 3. In our previous work the correlation was derived using 108 PAH, containing 493 symmetry unique rings. In this study a much larger set of $286 \mathrm{PAH}$, containing 2640 symmetry unique rings is used. This larger test set reveals that the results of NICS and the MCBI for some specific rings can not be reconciled using this method. We show that the problem lies in the non-local character of the NICS. Furthermore the results prove that including this non-local character of the NICS in the model, using the MCBI-vector maps mentioned above, explains the difference between the NICS and MCBI 
for the problematic rings. The authors also examined whether or not circuits other than the Benzene, Naphthalene, Anthracene- and Phenanthrene-like have a significant effect on the NICS.

\section{Computational Methods}

The correlation between MCBI and NICS was derived using a set of $108 \mathrm{PAH}$, built from one to six benzenoid rings. The correlation was subsequently evaluated using a test set of 286 PAH built from seven and eight benzenoid rings. All molecular geometries were constructed using the Benzene geometry with a C-C bond length of 1.4 $\AA$. The MCBI for all these molecules were calculated using the Pseudo- $\pi$ approach ${ }^{[1,28-30]}$. The MCBI were computed using the in home developed Kekule program which uses the Gaussian-03 ${ }^{[31]}$ formatted

checkpoint file. The NICSzz ${ }^{[10,32]}$ were also calculated in the Pseudo- $\pi$ approach $^{[1]}$ using Gaussian-03. For the study of correlations between the NICS and multicenter indices, the ARTE-QSAR program was used ${ }^{[33]}$.

\section{Results and discussion}

Using the constants a to d in equation 3, obtained from a set of $108 \mathrm{PAH}$, the $\mathrm{NICS}_{\mathrm{zz}}$ of the test set of $286 \mathrm{PAH}$ were predicted using the same equation. The NICS $_{\mathrm{zz}}$ versus the Predicted-NICS $\mathrm{zz}$ for all 394 molecules of the fitting and test set together are shown in Figure 1.

The majority of the rings is well predicted and the $\mathrm{R}^{2}$ is 0.884 , however the method fails to predict some rings with positive NICS values. The NICS of the cluster of points in the upper right corner (green) in figure 1 is pre- 
dicted too low. These points correspond to the central ring of Perylene-like fragments in the PAH (Figure 2(a)). The points in the lower right quadrant (orange) are predicted with the wrong sign. These points correspond to the central rings of Benzo[ghi]perylene fragments in the molecules (Figure 2(b)). The red point corresponds to the central ring of corenene (Figure 2(c)) whose NICS is dramatically underestimated using the method. The poor prediction of the NICS for these rings using this model is in contrast to the fact that the MCBI-ring current maps of these molecules are indistinguishable from the $a b$ initio Ring Current calculations on these molecules ${ }^{[22]}$. The SCI values of the individual rings reveal the reason for the poor agreement with the NICS. In all these cases the central ring is surrounded by benzenoid rings with a much higher six centre delocalization (Figure 2). In Perylene the delocalisation in the cental ring is very small, whereas the two top and bottom rings have higher delocalisation values. In the ring current and MCBI-RC maps this results two distinct Naphthalene-like circuits and the current on the bonds connecting these two Naphthalene substructures is essentially zero ${ }^{[22]}$. The NICS of the inner ring is positive, not because of the delocalisation in this central ring, but because of the higher delocalisation in the neighbouring rings. The NICS of the inner ring is induced by the Naphthalene-like circuits above and below the central ring, but it is essentially an empty ring, flanked by two diatropic Naphthalene circuits. Similarly in Benzo[ghi]perylene and Corenene the paratropic current in the central ring is a consequence of the difference in benzenoid delocalization between the central and the outer rings, where the outher rings overpower the inner one.

With this observation in mind, one can attempt to prove the existance 
of these non-local effects on the NICS statistically by changing the model in such way that it takes these effects into account. This can be done by constructing the SCI, TCI and FCI vector maps as described before(Figure $3 \mathrm{a}$ and $3 \mathrm{~b}$ ) and by summing the values of the vectors around the benzenoid ring to a new SCI, TCI or FCI value for each ring . This is done by summing the SCI,TCI of FCI values of the vectors which run in a diatropic manner around the benzenoid ring and by substracting the values of the paratropic vectors of the ring(Figure 3c). These new SCI, TCI and FCI values are now corrected for the effect of (partial) cancellation that the delocalisations in the neighbouring rings have on the current density. Introducing these 'corrected' SCI, TCI and FCI in equation 3 should result in a closer correlation with the NICS values. The constants a to $d$ where again obtained using the fitting set of $108 \mathrm{PAH}$ and subsequently tested on the test set of $286 \mathrm{PAH}$. The $\mathrm{NICS}_{\mathrm{zz}}$ versus the predicted-NICS $\mathrm{zz}$ using this method for all 394 molecules are shown in Figure 4.

Using this method, which will be called the vector method from hereon, the $\mathrm{R}^{2}$ increases from 0.88 to 0.94 . Moreover the central rings of Benzo[ghi]perylene fragments (orange) have the right sign and the central ring of Perylene-like fragments (green) are better predicted. These findings show that the vector-model predicts the NICS better than the much simpeler model of equation 3. This proves that the NICS value is not only influenced by the higher-order circuits encircling the ring, but also by the local aromaticity of the surrounding rings. The NICS for the central ring of corenene however changes from underestimated to overestimated, with a predicted value of $16.60 \mathrm{ppm}$ instead of the ab-initio $-0.60 \mathrm{ppm}$. 
To get a better agreement between the MCBI-predicted NICS and the abinitio NICS, one possibly needs to consider more delocalisation circuits. The current model only uses Benzene-, Naphthalene, Anthracene- and Phenanthrenelike indices. However many of the PAH also contain phenalene-like twelvecentre circuits and pyrene-like fourteen-centre circuits which are not accounted for in the models described above, but might contribute to the NICS in a similar fashion. To see whether these twelve- and fourteen-centre circuits have an effect on the NICS, the indices were calculated on all molecules and the above vector-model was reconstructed using a six, ten-, twelve-, and fourteen-centre vector map, where the pyrene-like circuit was summed together with the Anthracene- and Phenanthrene-circuits to one fourteencentre vector map. This summation to one map is based on the previous finding that separating the Anthracene- and Phenanthrene-circuits does not improve the model significantly ${ }^{[1]}$. We wish to avoid introducing too many variables, resulting in more flexibility in the model than can physically be justified. Inclusion of even higher-order circuits could also be considered, however since it has been shown that the influence of the circuit becomes smaller with increasing numer of centres ${ }^{[1]}$ and keeping the increasing computational cost in mind, only circuits up to fourtheen centres were considered in this study.

The results for the vector-method including the phenalene-like twelveand pyrene-like fourteen-centre circuits are shown in Figure 5. These results are again obtained by fitting the model $108 \mathrm{PAH}$ and subsequently using the model for the set of $286 \mathrm{PAH}$. The immediate observation is that the introduction of the two extra circuits does not change the previous findings. 
There is no drastic change in the correlation or in the appearence of the graph. The NICS for the central ring of Coronene remains overestimated, although there is a small correction in the right direction from $16.60 \mathrm{ppm}$ to $12.03 \mathrm{ppm}$. The introduction of the twelve-centre vector map is however statistically verified as significant. The t-test reveals a $99.95 \%$ certainty that the twelvecentre vector map has a significant contibution. The small effect on the results is primarily due to the absence of a twelve-centre circuit in many of the PAH and the small effect the circuit has on the total NICS. The average weight of the twelve-centre ciruit for the total NICS value is only $2.1 \%$. An interesting observation is that the twelve-centre delocalisation contibutes in a paratropic fashion to the NICS, wereas all other circuits give diatropic contributions. This suggests that the $4 \mathrm{~N}+2$ rule is also applicable to the delocalisation indices, where $(4 \mathrm{~N}+2)$ - and $4 \mathrm{~N}$-membered circuits contribute diatropic and paratropic, respectively.

Turning back to corenene, the current adaptations to the model fail to discribe the NICS of the central ring, although it has already been shown that the MCBI-ring current map of this molecule is indistinguishable from the full Ring Current calculation ${ }^{[22]}$. The MCBI-ring current map of Coronene (Figure 4) reveals the possible problem. The paratropic current on the central ring is rather small compared to the outer diatropic current. In the vectormethod used until now, only the vectors on the benzenoid ring at wich the NICS is evaluated are used, while other vectors farther from the ring are neglected. Using the continous set of gauge transformations ${ }^{[34-36]}$, the effect of the ring current $(\mathbf{J}(\mathbf{r}))$ to the zz-component of the shielding tensor $\left(\sigma^{z z}\left(\mathbf{r}_{X}\right)\right)$ is proportional to $\times \mathbf{r}_{X} / r^{3}$ : 


$$
\sigma^{z z}\left(\mathbf{r}_{X}\right)=-\frac{1}{B c} \int\left\{\left(\mathbf{r}-\mathbf{r}_{X}\right) \times \mathbf{J}_{Z}^{(1)}(\mathbf{r})\right\}_{z} / r^{3} d \mathbf{r}
$$

Where $\mathbf{J}_{Z}^{(1)}(\mathbf{r})$ the first-order electronic current density induced by a magnetic field along the $\mathrm{Z}$ axis. The effect of vectors farther from the ring thus diminishes roughly by $1 / r^{2}$, which justifies neglecting the vectors farther from the ring. In the case of Coronene however, the outer current is so strong it can not be neglected for calculating the NICS of the central ring. The fact that only the vectors on the central ring were used is responsible for overestimating the NICS value. The answer to the correct prediction of the central ring of Coronene might thus lie in including all vectors of the molecule into the model.

Since the total ring current can be considered as composed of contributons from different circuits ${ }^{[24-26]}$ (equation 1) and as the contribution from a given $\mathrm{N}$-centre circuit $(\mathrm{NC})$ to the current density $\left(\mathbf{J}_{Z}(\mathbf{r} ; N C)\right)$ is proportional to the $\mathrm{MCBI}$ for the circuit $\mathrm{NC}, \mathbf{J}_{Z}(\mathbf{A B} ; N C)$ can be approximated by the MCBI projected as a vector in a diatropic manner on the bond between atoms $\mathrm{A}$ and $\mathrm{B}(\mathbf{M C B I}(\mathbf{A B} ; N C))$, thus:

$$
\mathbf{J}_{Z}(\mathbf{A B} ; N C) \sim \operatorname{MCBI}(\mathbf{A B} ; N C)
$$

The total ring current can be written in terms of the MCBI-vectors as: 


$$
\begin{aligned}
\mathbf{J}_{Z}^{(1)}(A B) & =a \sum_{N S C I} \mathbf{S C I}(\mathbf{A B} ; N S C I) \\
& +b \sum_{N T C I} \mathbf{T C I}(\mathbf{A B} ; N T C I) \\
& +c \sum_{N 12 C I} \mathbf{1 2 C I}(\mathbf{A B} ; N 12 C I) \\
& +d \sum_{N F C I} \mathbf{F C I}(\mathbf{A B} ; N F C I) \\
& +e
\end{aligned}
$$

Similarly the expression for the NICS can be rewritten in terms of shielding tensors associated to the vector map of a certain delocalisation orde $\left(\sigma^{z z}\left(\mathbf{r}_{X} ; M C B I\right)\right)$ :

$$
\begin{aligned}
\operatorname{NICS} z z\left(\mathbf{r}_{\mathbf{x}}\right)=- & \left(a \sigma^{z z}\left(\mathbf{r}_{X} ; S C I\right)+b \sigma^{z z}\left(\mathbf{r}_{X} ; T C I\right)+c \sigma^{z z}\left(\mathbf{r}_{X} ; 12 C I\right)\right. \\
& \left.+d \sigma^{z z}\left(\mathbf{r}_{X} ; F C I\right)+e\right)
\end{aligned}
$$

Where the component of the shielding tensor associated to a certain delocalisation order $\left(\sigma^{z z}\left(\mathbf{r}_{X} ; M C B I\right)\right)$ can be written as:

$\sigma_{X}^{z z}\left(\mathbf{r}_{X} ; M C B I\right)=-\frac{1}{B C} \sum_{A B=1}^{N}\left\{\left(\mathbf{r}_{\overline{A B}}-\mathbf{r}_{X}\right) \times \sum_{N C=1}^{M} \operatorname{MCBI}(\mathbf{A B} ; N C)\right\}_{z} / r_{X, \overline{A B}}^{3}$

The summations run over all the bonds (AB) and all the different circuits with a given number of centres (NC) respectivly.

Constants a, b, c, and d from equation 7 can now be obtained by calculating the shielding tensors from the vector maps of the different delocalisation 
circuits (Equation 8). This slightly more complex procedure should correctly describe the NICS of the inner ring of Coronene. The results for this method are represented in Figure 7. Once again the model was fitted for the 108 PAH and subsequently used for the large set of $286 \mathrm{PAH}$.

The figure shows that this model indeed closely predicts the NICS of the inner ring of Coronene, which is now predicted as $3.26 \mathrm{ppm}$ (the ab initio value is -0.60$)$. The $R^{2}$ does not increase signifficantly (0.947 to 0.950 ) and the rooth mean square decreases only a little, from 2.55 to 2.45 . The model is not necessarily better in describing the NICS for the whole set of molecules, compared to the simpler vector-model. This is because the $\times \mathbf{r}_{X} / r^{3}$ dependence ensures that the effect of other vectors than the ones encircling the ring at which the NICS is evaluated can be neglected in the majority of the PAH. The model is however necessary to correctly understand the NICS in Coronene. The example of Coronene nicely demonstrates that the NICS computed at a benzenoid ring center should not be used to compare the local aromaticity of benzenoid circuits. In this molecule not only do the different circuits contribute to the NICS value, but the NICS of the central ring is a result of the more aromatic Benzene circuits surrounding the inner ring. The effect is subsequently reduced by the large diatropic current running around the edge of the molecule.

The apparent multidimensional character is thus shown to be fictious. Multicentre indices, used to assess benzenoid character, are strictly related to the benzenoid ring and no direct effect of the neighboring rings is considered. This allows considering local benzenoid aromaticity as the retention of similarity of a benzenoid ring to isolated Benzene. NICS take a entirely 
different approach and reflect in a single point all the currents in a molecule. In the case of PAH, local aromaticity is not multidimensional, it simply follows a different definition. The lack of need for multidimensionality is clearly illustrated by the fact that both can be reconciled as shown above.

\section{Conclusions}

In this paper the non-local effect on the NICS were studied using the MCBI for a large set of 394 PAH. This revealed that for Perylene-like and Benzo[ghi]perylene-like fragments and for the central ring of Coronene, The NICS value can not be explained using the six-, ten- and fourteen-centre circuits alone as in the previous study ${ }^{[1]}$. It was shown that the problem lies in the non-local character of the NICS. This non-local character could be proven using the MCBI-vector maps. Furthermore it was shown that phenalene like twelvecentre circuits and pyrene-like fourteen-centre circuits at first glance do not lead to a better correlation between the NICS and the MCBI, but statistics show the effect on the NICS is meaningful. The models presented here do not serve as an alternative way to calculate the NICS using the MCBI, but were constructed to fully examine and understand the difference between the NICS and delocalisation indices. In this way, these results have further demonstrated that NICS should not be used to assess the degree of local aromaticity of a benzenoid circuit as MCBI do. Three different non-local efects on the NICS were found:

- the influence of the higher-order circuits encircling the ring. Not only the six-, ten- and fourteen-centre circuits have an effect on the NICS, 
the present study show that also the twelve-centre circuits have a meaningful influence.

- the influence of local aromaticity of the surrounding, neighbouring, rings which can dramatically change the current on the commun bonds of adjoint rings, influencing the NICS of these rings.

- the influence of currents even farther away from the ring at which the NICS is evaluated, like the effect of the outer current in Coronene on the NICS in the central ring.

These results show it is possible to find a very tight correlation between the MCBI and the NICS, when taking suitable combination of the delocalisation indices for different circuits. These results confirm once more that the claimed multidimensionality of aromaticity must not be involved in this case. Scrutiny must be used when reporting correlations between aromaticity indices reflecting only a specific circuit and NICS values.

While examining the non-local contributions to the NICS, the results of this study also raised the suggestion that there is a $4 \mathrm{~N}+2$ rule applicable to the delocalisation indices, where $(4 \mathrm{~N}+2)$ - and $4 \mathrm{~N}$-membered circuits contribute in a diatropic and paratropic manner, respectively. However, further investigation of this $4 \mathrm{~N}+2$ rule for delocalisarion indices is necessary.

\section{Acknowledgments}

PB wishes to thank Ghent University and the Fund for Scientific ResearchFlanders (Belgium) for their grants to the Quantum Chemistry group at Ghent University. The present research is partially funded by a $\mathrm{PhD}$. grant 
to SVD of the Institute for the Promotion of Innovation through Science and Technology in Flanders (IWT Vlaanderen).

\section{References}

[1] Fias, S.; Van Damme, S.; Bultinck, P. J. Comput. Chem. 2008, 29, $358-366$.

[2] Kekulé, A. Lehrbuch der Organischen Chemie, Zweiter Band; Ferdinand Enke Verlag, Erlangen, 1866.

[3] Cyrañski, M.; Krygowski, T. M.; Katritzky, A. R.; Schleyer, P. v. R. J. Org. Chem. 2002, 67, 1333-1338.

[4] Krygowski, T.; Cyrañski, M. Chem. Rev. 2001, 101, 1385-1419.

[5] Poater, J.; Duran, M.; Solà, M.; Silvi, B. Chem. Rev. 2005, 105, 39113947.

[6] Elser, V.; Haddon, R. Nature 1987, 325, 792-794.

[7] Schleyer, P.; Maerker, C.; Dransfeld, A.; Jiao, H.; Hommes, N. J. Am. Chem. Soc 1996, 118, 6317-6318.

[8] Lazzeretti, P. Phys. Chem. Chem. Phys. 2004, 6, 217-223.

[9] Steiner, E.; Fowler, P. W. J. Phys. Chem. A 2001, 105, 9553-9562.

[10] Steiner, E.; Fowler, P.; Jenneskens, L. Angew. Chem. Int. Ed. 2001, 40, $362-366$. 
[11] Bultinck, P.; Ponec, R.; Van Damme, S. J. Phys. Org. Chem. 2005, 18, 706-718.

[12] Bultinck, P.; Rafat, M.; Ponec, R.; Carbó-Dorca, R.; Popelier, P. J. Phys. Chem. A 2006, 110(24), 7642-7648.

[13] Bultinck, P.; Fias, S.; Ponec, R. Chem.-Eur. J. 2006, 12, 8813-8818.

[14] Bultinck, P.; Ponec, R.; Carbó-Dorca, R. J. Comput. Chem. 2007, 28, 152-160.

[15] Feixas, F.; Matito, E.; Poater, J.; Solà, M. J. Comput. Chem. 2008, 29(10), 1543-1554.

[16] Schleyer, P. v. R.; Jiao, H.; Hommes, N. J. R. v. E.; Malkin, V. G.; Malkina, O. J. Am. Chem. Soc. 1997, 119, 12669-12670.

[17] Fallah-Bagher-Shaidaei, H.; Wannere, C.; Corminboeuf, C.; Puchta, R.; Schleyer, P. Org. Lett. 2006, 8, 863-866.

[18] Jug, K.; Koster, A. M. J. Phys. Org. Chem. 1991, 4, 163-169.

[19] Katritzky, A. R.; Karelson, M.; Sild, S.; Krygowski, T. M.; Jug, K. J. Org. Chem. 1998, 63, 5228-5231.

[20] Katritzky, A. R.; Barczynski, P.; Musumarra, G.; Pisano, D.; Szafran, M. J. Am. Chem. Soc. 1989, 111, 7-15.

[21] Poater, J.; García-Cruz, I.; Illas, F.; Solà, M. Phys. Chem. Chem. Phys. 2004, 6, 314-318.

[22] Fias, S.; Fowler, P. W.; Delgado, J. L.; Hahn, U.; Bultinck, P. Chem. Eur. J. 2008, 14, 3093-3099. 
[23] Havenith, R. W. A.; Fowler, P. W.; Fias, S.; Bultinck, P. Tet. Lett. 2008, 49, 14211424.

[24] Aihara, J. Chem. Phys. Lett. 2004, 393, 7-11.

[25] Aihara, J.; Kanno, H. J. Phys. Chem. A 2005, 109, 3717-3721.

[26] Aihara, J. J. Am. Chem. Soc. 2006, 128, 2873-2879.

[27] Schleyer, P. v. R.; Manoharan, M.; Wang, Z.-X.; Kiran, B.; Jiao, H.; Puchta, R.; Hommes, N. E. Org. Lett. 2001, 3, 2465-2468.

[28] London, F. J. Phys. Radium 1937, 8, 397.

[29] Fowler, P. W.; Steiner, E. Chem. Phys. Lett. 2002, 364, 259-266.

[30] Bultinck, P.; Mandado, M.; Mosquera, R. J. Math. Chem. 2007, 43, $111-118$.

[31] Gaussian 03, revision b.03. Frisch, M. J.; Trucks, G. W.; Schlegel, H. B.; Scuseria, G. E.; Robb, M. A.; Cheeseman, J. R.; Montgomery, J. A.; Vreven, T.; Kudin, K. N.; Burant, J. C.; Millam, J. M.; Iyengar, S. S.; Tomasi, J.; Barone, V.; Mennucci, B.; Cossi, M.; Scalmani, G.; Rega, N.; Petersson, G. A.; Nakatsuji, H.; Hada, M.; Ehara, M.; Toyota, K.; Fukuda, R.; Hasegawa, J.; Ishida, M.; Nakajima, T.; Honda, Y.; Kitao, O.; Nakai, H.; Klene, M.; Li, X.; Knox, J. E.; Hratchian, H. P.; Cross, J. B.; Adamo, C.; Jaramillo, J.; Gomperts, R.; Stratmann, R. E.; Yazyev, O.; Austin, A. J.; Cammi, R.; Pomelli, C.; Ochterski, J. W.; Ayala, P. Y.; Morokuma, K.; Voth, G. A.; Salvador, P.; Dannenberg, J. J.; Zakrzewski, V. G.; Dapprich, S.; Daniels, A. D.; Strain, M. C.; 
Farkas, O.; Malick, D. K.; Rabuck, A. D.; Raghavachari, K.; Foresman, J. B.; Ortiz, J. V.; Cui, Q.; Baboul, A. G.; Clifford, S.; Cioslowski, J.; Stefanov, B. B.; Liu, G.; Liashenko, A.; Piskorz, P.; Komaromi, I.; Martin, R. L.; Fox, D. J.; Keith, T.; Al-Laham, M. A.; Peng, C. Y.; Nanayakkara, A.; Challacombe, M.; Gill, P. M. W.; Johnson, B.; Chen, W.; Wong, M. W.; Gonzalez, C.; Pople, J. A. 2003.

[32] Fowler, P.; Steiner, E. Mol. Phys. 2000, 98, 945-953.

[33] Van Damme, S.; Bultinck, P. J. Comput. Chem. 2007, 28(11), 19241928.

[34] Keith, T. A.; Barder, R. F. W. Chem. Phys. Lett. 1992, 194, 1-8.

[35] Keith, T. A.; Barder, R. F. W. Chem. Phys. Lett. 1993, 210, 223-231.

[36] Keith, T. A.; Barder, R. F. W. J. Chem. Phys. 1993, 99, 3669-3682. 


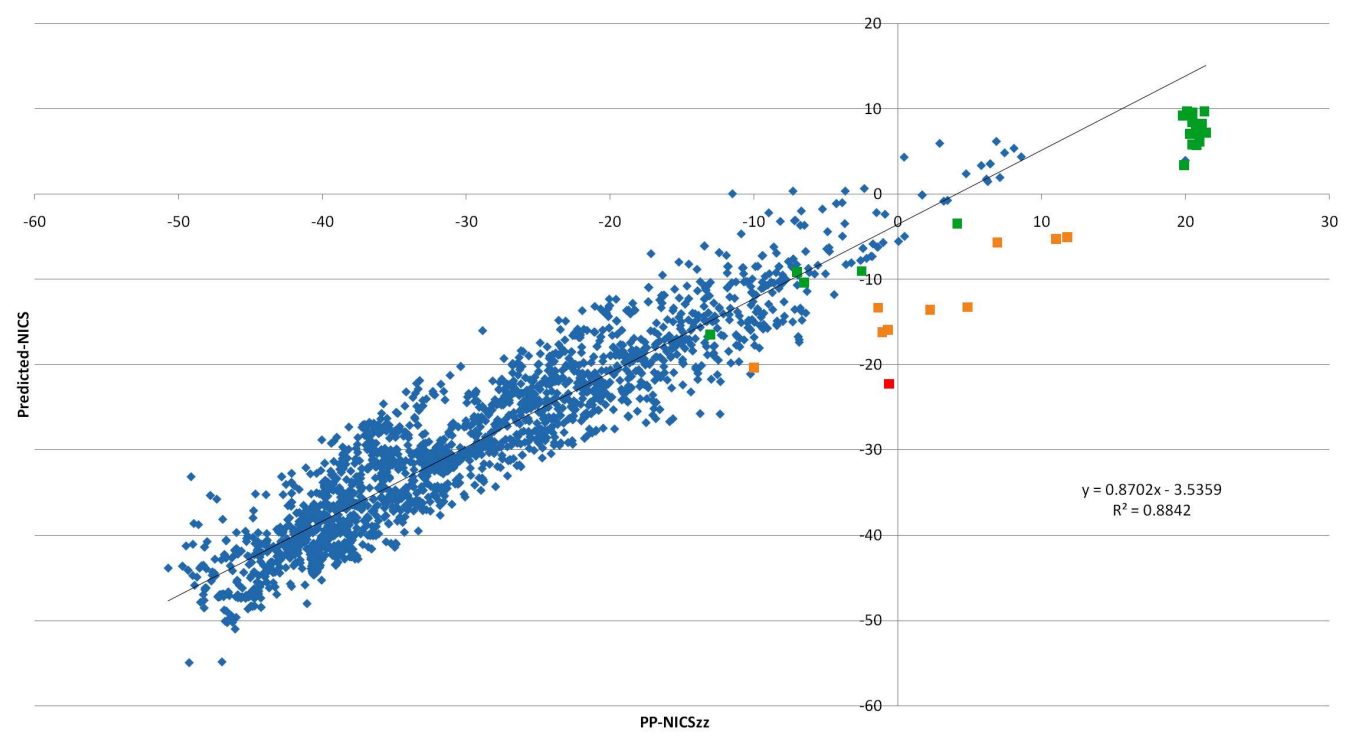

Figure 1: The $\mathrm{NICS}_{\mathrm{zz}}$ versus the Predicted-NICS $\mathrm{zz}$ for $394 \mathrm{PAH}$ using equation 3

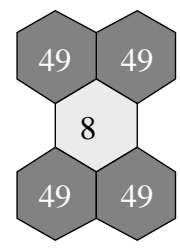

(a)

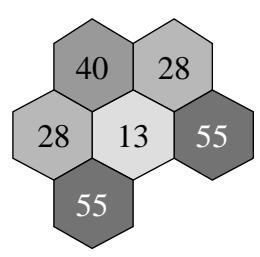

(b)

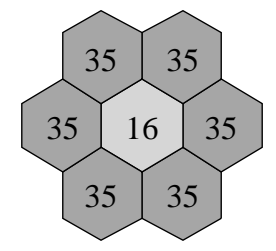

(c)

Figure 2: SCI values for Perylene(a), Benzo[ghi]perylene(b) and Coronene(c), expressed in percentages of the Benzene value 


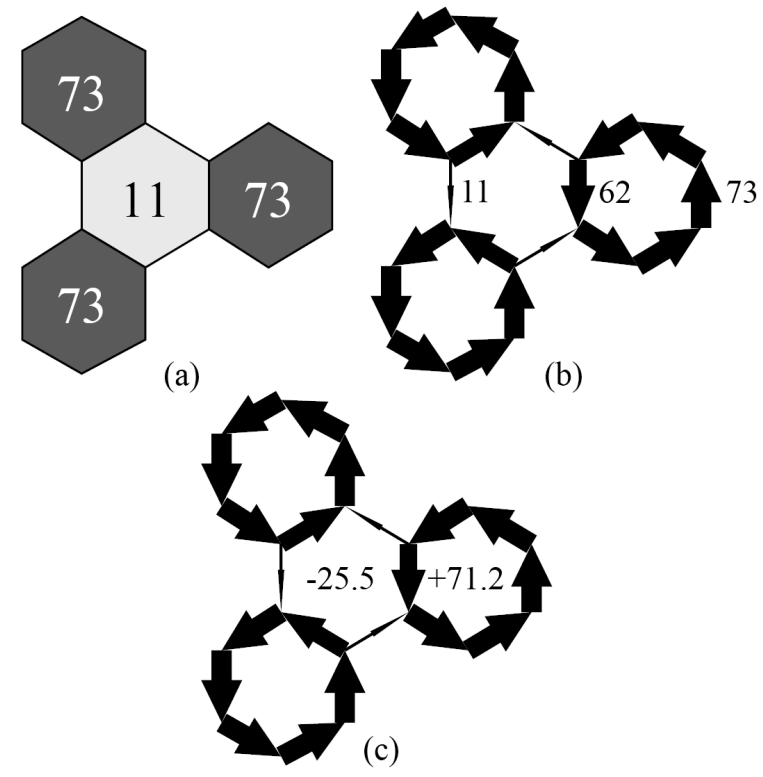

Figure 3: The construction of the new SCI values using the vector method of triphenylene. (a) The values of the SCI of the rings, expressed in terms of the percentages of Benzene value. (b) The SCI projected as vectors on the bonds of the benzenoid ring in a diatropic manner. The SCI values of the adjoint rings partially cancel each other on the common bonds (c) The values of the vectors running in a diatropic manner around the benzenoid ring are summed, the paratropic vectors are substracted and the value is divided by six to form the new SCI. 


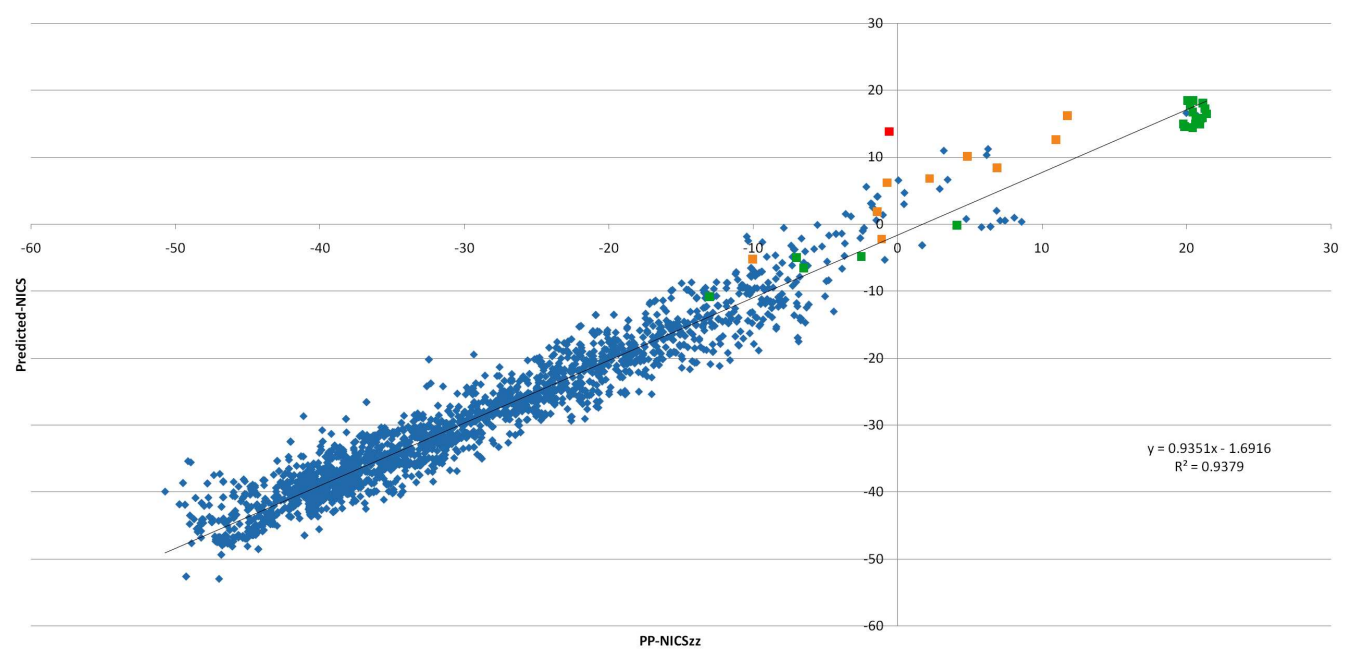

Figure 4: The NICS$_{\mathrm{zz}}$ versus the Predicted-NICS $\mathrm{zz}$ for $394 \mathrm{PAH}$ using the vector method

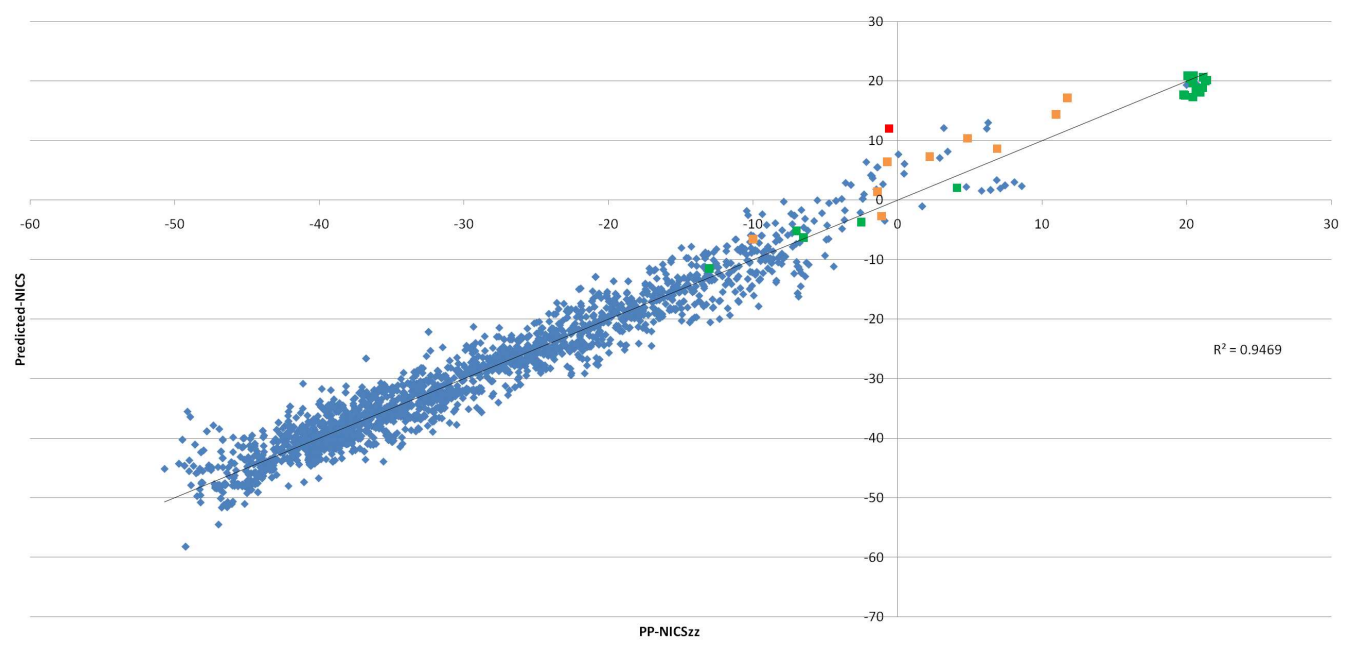

Figure 5: The NICS$_{\mathrm{zz}}$ versus the Predicted-NICS $\mathrm{zz}$ for $394 \mathrm{PAH}$ using the vector method, including the Phenalene-like twelve-centre and Pyrene-like fourteen-centre circuits 


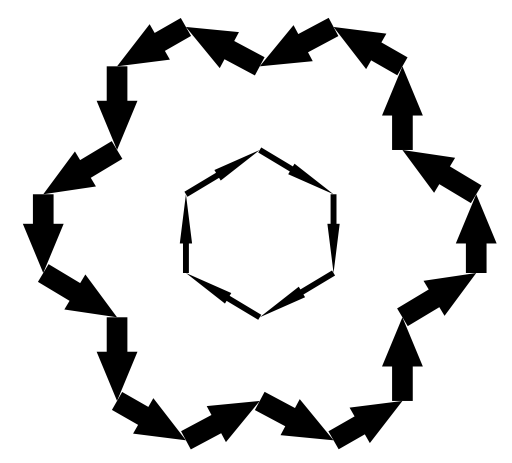

Figure 6: The MCBI-ring current map of Coronene.

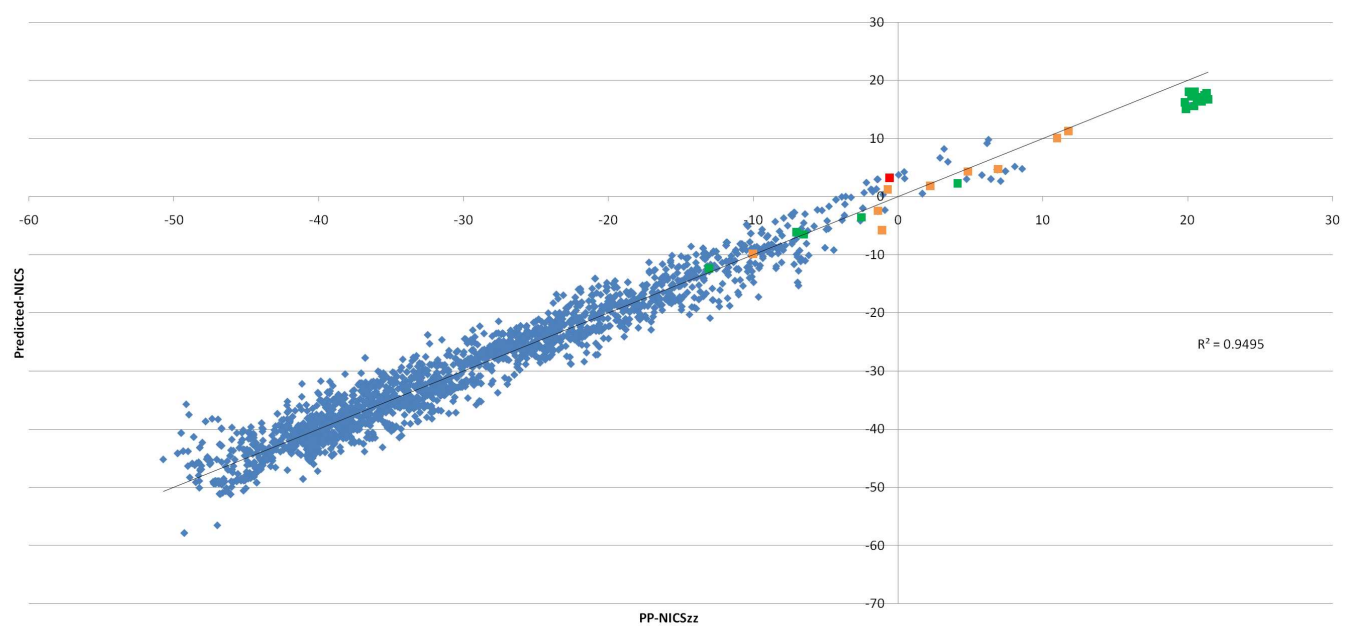

Figure 7: The NICS $_{z z}$ versus the Predicted-NICS ${ }_{\mathrm{zz}}$ for $394 \mathrm{PAH}$ using equation 7 